J. Nonlinear Var. Anal. 6 (2022), No. 1, pp. 149-163

Available online at http://jnva.biemdas.com

https://doi.org/10.23952/jnva.6.2022.1.09

\title{
ON GENERALIZED GLOBAL FRACTIONAL-ORDER COMPOSITE DYNAMICAL SYSTEMS WITH SET-VALUED PERTURBATIONS
}

\author{
LU-CHUAN CENG ${ }^{1}$, NAN-JING HUANG ${ }^{2}$, CHING-FENG WEN ${ }^{3,4, *}$ \\ ${ }^{1}$ Department of Mathematics, Shanghai Normal University, Shanghai 200234, China \\ ${ }^{2}$ Department of Mathematics, Sichuan University, Chengdu 610064, China \\ ${ }^{3}$ Center for Fundamental Science, and Research Center for Nonlinear Analysis and Optimization, \\ Kaohsiung Medical University, Kaohsiung 80708, Taiwan \\ ${ }^{4}$ Department of Medical Research, Kaohsiung Medical University Hospital, Kaohsiung 80708, Taiwan
}

\begin{abstract}
In this paper, we investigate a class of generalized global fractional-order composite dynamical systems involving set-valued perturbations in real separable Hilbert spaces. First, we prove that the solution set of the systems is nonempty and closed under some suitable conditions. Second, we show that the solution set is continuous with respect to the initial value in the sense of the Hausdorff metric. Last, an example is provided to illustrate the applicability of the main results.
\end{abstract}

Keywords. Fractional-order composite dynamical system; Set-valued perturbation; Sensitivity.

\section{INTRODUCTION}

In 1993, Dupuis and Nagurney [1] revealed the connection between a projective dynamical system and an associated variational inequality via the following local projective dynamical system

$$
\frac{d x}{d t}=\lim _{\rho \rightarrow 0} \frac{P_{K}(x-\rho N(x))-x}{\rho} .
$$

Subsequently, Friesze et al. [2] extended this type of systems to global ones and applied them to the problems in traffic network equilibrium analysis. Meanwhile, they showed that the tatonnement model of a certain traffic problem can be formulated as a simultaneous projective dynamical system

$$
\left\{\begin{aligned}
\frac{d h(t)}{d t} & =\eta\left\{P_{K}(h(t)-\rho \operatorname{ETC}(h(t), u(t)))-h(t)\right\}, & & \forall t \in[0, b], \\
\frac{d u(t)}{d t} & =\kappa\left\{P_{K}(u(t)+\lambda \operatorname{ETD}(u(t), h(t)))-u(t)\right\}, & & \forall t \in[0, b], \\
h(0) & =h_{0} \text { and } u(0)=u_{0}, & &
\end{aligned}\right.
$$

where $\rho, \lambda \in \mathbb{R}_{+}^{1}=[0, \infty)$.

\footnotetext{
${ }^{*}$ Corresponding author.

E-mail addresses: zenglc@ hotmail.com (L.-C. Ceng), nanjinghuang@hotmail.com; njhuang@scu.edu.cn (N.-J. Huang), cfwen@kmu.edu.tw (C.-F. Wen).

Received October 16, 2021; Accepted December 27, 2021.
}

(C)2022 Journal of Nonlinear and Variational Analysis 
Projective dynamical systems have been under the spotlight of research for the past decades. Both in-depth theories and wide applications of the projective dynamical systems have been studied extensively. For example, we refer the reader to [1-10] and the references therein.

To the best of our knowledge, many real life scientific or engineering problems are neither linear nor nonlinear, which makes it inappropriate to model them with differential systems of integer order. Instead, fractional derivatives provide a better tool than integer order ones to describe many physical processes, especially those with memory and hereditary properties; , see, e.g., [11-13]. Recently, some new results on fractional order differential systems have appeared in this field; see, e.g., [14, 15]. In particular, in 1984, Torvik and Bagley [11] showed that the fractional order model was effective in describing the behavior of real material. In 2011, Li and Zhang [12] did a survey on the stability of fractional differential equations. In 2012, Ozalp and Koca [13] introduced a fractional order dynamical model of interpersonal relationships. In 2015, Buyukkilic et al. [14] investigated the cumulative growth of a physical quantity via the Fibonacci method and fractional calculus.

On the other hand, $\mathrm{Wu}$ and Zou [16], for the first time, proposed the following global fractional-order projective dynamical system in $\mathbb{R}^{n}$

$$
\left\{\begin{array}{l}
{ }_{0}^{C} D_{t}^{\alpha} x(t)=P_{K}(x(t)-\rho M x(t)-\rho q)-x(t), \quad t \geq 0, \\
x_{i}(0)=x_{i, 0}, \quad i=1,2, \ldots, n,
\end{array}\right.
$$

where $0<\alpha<1$, and $M$ is a real $n \times n$ matrix. Then the existence and uniqueness of solutions and the existence of equilibrium points were obtained, and they showed that equilibrium points were $\alpha$-exponentially stable.

Recently, Wu et al. [17] considered a class of fractional set-valued projected dynamical systems in $\mathbb{R}^{n}$

$$
\left\{\begin{array}{l}
{ }_{0}^{C} D_{t}^{\alpha} x(t) \in P_{K}(g(x(t))-\lambda N(x(t)))-g(x(t)), \quad \text { for } t \in[0, b] \\
x(0)=p, x(b)=q, \quad p, q \neq 0,
\end{array}\right.
$$

where $\alpha \in[1,2)$, and $N: \mathbb{R}^{n} \rightarrow 2^{\mathbb{R}^{n}}$ is a set-valued mapping. The nonemptiness and closedness of the solution set were obtained, and they showed that the solution set was continuous with respect to the boundary value in the sense of the Hausdorff metric.

In 2016, based on Wardropian user equilibrium tatonnement model, Wu et al. [18] investigated a class of fractional-order interval projective dynamical systems in $\mathbb{R}^{n} \times \mathbb{R}^{m}$

$$
\left\{\begin{array}{l}
{ }_{0}^{C} D_{t}^{\alpha} x(t)=P_{K_{1}}\left[x(t)-\rho\left(A x(t)+A^{*} y(t)\right)-\rho p\right]-x(t), t \geq 0, \\
{ }_{0}^{C} D_{t}^{\alpha} y(t)=P_{K_{2}}\left[y(t)-\lambda\left(B y(t)+B^{*} x(t)\right)-\lambda q\right]-y(t), t \geq 0, \\
x(0)=x_{0} \text { and } y(0)=y_{0}
\end{array}\right.
$$

where $0<\alpha \leq 1$ and

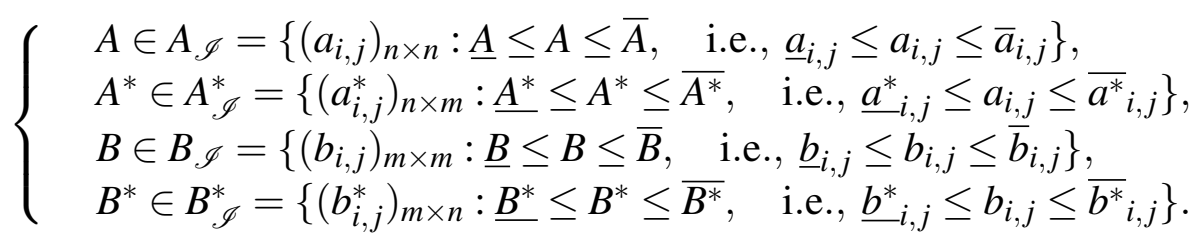

They proved the existence ant uniqueness of the equilibrium point under some suitable conditions and obtained the $\alpha$-exponential stability of this type of projective dynamical systems.

It is well known that, for the global fractional-order projective dynamical systems in abstract spaces, there are little few results in the existing literature. Very recently, Wu et al. [3] made an 
attempt in this direction. Moreover, the system may appear perturbation by external factor. In particular, the perturbation is a set-valued mapping. In [3], the following fractional projective dynamical system with set-valued perturbations was first introduced in real separable Hilbert spaces:

$$
\begin{cases}{ }_{0}^{C} D_{t}^{\alpha} x(t) \in P_{K_{1}}(x(t)-\rho M(x(t), y(t))-\rho p)-x(t)+G_{1}(x(t)), & \text { for a.e. } t \in[0, b], \\ { }_{0}^{C} D_{t}^{\alpha} y(t) \in P_{K_{2}}(y(t)-\lambda N(y(t), x(t))-\lambda q)-y(t)+G_{2}(y(t)), & \text { for a.e. } t \in[0, b], \\ x(0)=x_{0} \text { and } y(0)=y_{0}, & \end{cases}
$$

where ${ }_{0}^{C} D_{t}^{\alpha}$ is the Caputo fractional derivative of order $\alpha \in(0,1], P_{K_{1}}$, and $P_{K_{2}}$ are two projection operators, $K_{1}$ and $K_{2}$ are two nonempty closed convex subsets of two separable Hilbert spaces $V_{1}$ and $V_{2}$, respectively, $\rho>0$ and $\lambda>0$ are two constants, $x, x_{0}, p \in V_{1}, y, y_{0}, q \in V_{2}, M: V_{1} \times V_{2} \rightarrow$ $V_{1}$, and $N: V_{2} \times V_{1} \rightarrow V_{2}$ are two nonlinear mappings, and $G_{1}: V_{1} \rightarrow 2^{V_{1}}$ and $G_{2}: V_{2} \rightarrow 2^{V_{2}}$ are two set-valued mappings. Obviously, in Euclidean spaces, if $G_{1}=G_{2}=0, M(x(t), y(t))=$ $A x(t)+A^{*} y(t)$, and $N(y(t), x(t))=B y(t)+B^{*} x(t)$, then (1.4) reduces to (1.3). If $G_{1}=G_{2}=$ $0, N=0, q=0, y=0, \alpha \in(0,1)$, and $M$ is a real matrix, then (1.4) reduces to (1.1). In addition, if we consider the two point boundary value problem, $\alpha \in[1,2), M$ is a set-valued mapping, then (1.4) reduces to (1.2). It is worth mentioning that if $K_{1}=V_{1}$ and $K_{2}=V_{2}$, then (1.4) reduces to a system of two related fractional differential inclusions.

Let $\alpha \in(0,1], J=[0, b]$ for some $0<b<\infty$, and $V=V_{1} \times V_{2}$, where $V_{i}$ is a separable Hilbert space for $i=1,2$. Endowed with the norm defined by $\|\mathbf{x}\|_{V}:=\left\|x_{1}\right\|_{V_{1}}+\left\|x_{2}\right\|_{V_{2}}$ for all $\mathbf{x}=$ $\left(x_{1}, x_{2}\right) \in V, V$ is a reflexive Banach space (see e.g., [19]). Inspired by the above research works in [3], in this paper, we consider the following generalized fractional composite dynamical system with set-valued perturbations in real separable Hilbert spaces:

$$
\left\{\begin{array}{l}
{ }_{0}^{C} D_{t}^{\alpha} x_{1}(t) \in P_{1}\left(x_{1}(t)-\rho_{1} N_{1}\left(\Phi_{1}^{1}\left(x_{1}(t)\right), \Phi_{2}^{1}\left(x_{2}(t)\right)\right)-\rho_{1} q_{1}\right)-x_{1}(t)+G_{1}\left(x_{1}(t)\right), \text { a.e. } t \in J, \\
{ }_{0}^{C} D_{t}^{\alpha} x_{2}(t) \in P_{2}\left(x_{2}(t)-\rho_{2} N_{2}\left(\Phi_{1}^{2}\left(x_{1}(t)\right), \Phi_{2}^{2}\left(x_{2}(t)\right)\right)-\rho_{2} q_{2}\right)-x_{2}(t)+G_{2}\left(x_{2}(t)\right), \text { a.e. } t \in J, \\
x_{1}(0)=x_{1,0} \text { and } x_{2}(0)=x_{2,0},
\end{array}\right.
$$

where ${ }_{0}^{C} D_{t}^{\alpha}$ is the Caputo fractional derivative of order $\alpha \in(0,1]$. For $i=1,2, P_{i}: V_{i} \rightarrow V_{i}$ is nonexpansive, $\rho_{i}>0$ is a constant, $x_{i}, x_{i, 0}, q_{i} \in V_{i}, N_{i}: V_{1} \times V_{2} \rightarrow V_{i}$ is a nonlinear mapping, and $\Phi_{i}^{1}, \Phi_{i}^{2}, G_{i}: V_{i} \rightarrow 2^{V_{i}}$ all are set-valued mappings. In particular, if, for $i=1,2, P_{i}=P_{K_{i}}$ and $\Phi_{i}^{1}=\Phi_{i}^{2}=I$ the identity mapping of $V_{i}$, system (1.5) actually reduces to system (1.4).

The rest of this paper is organized below. In Section 2, we begin with some basic concepts and useful lemmas. In Section 3, the nonemptiness and closedness of the solution set for system (1.5) are first shown under some suitable conditions. It is also proven that the solution set is continuous with respect to the initial value in the sense of the Hausdorff metric. An example is provided to support our main results in Section 4. Finally, the concluding remark is given in Section 5.

\section{PRELIMINARIES}

In this section, we introduce some basic definitions and preliminaries which are used throughout this paper. We will denote the norm and dual space of a Banach space $X$ by $\|\cdot\|_{X}$ and $X^{*}$, respectively, and the duality pairing between $X^{*}$ and $X$ by $\langle\cdot, \cdot\rangle_{X}$. Let $C(J, X)$ be the Banach 
space of all continuous functions $x(t)$ from $J=[0, b]$ into $X$ with norm

$$
\|x\|_{C(J, X)}=\max _{t \in J}\|x(t)\|_{X}
$$

and let $L^{1}(J, X)$ be the Banach space of all Bochner integrable functions $x: J \rightarrow X$ with norm

$$
\|x\|_{L^{1}(J, X)}=\int_{0}^{b}\|x(t)\| d t .
$$

Let $\mathscr{C}(X)$ denote the family of all nonempty compact subsets of $X$, and let $\mathscr{H}(\cdot, \cdot)$ be the Hausdorff metric $\mathscr{H}(\cdot, \cdot)$ on $\mathscr{C}(X)$ defined by

$$
\mathscr{H}(A, B)=\max \left\{\sup _{y \in B} \inf _{x \in A} d(x, y), \sup _{x \in A} \inf _{y \in B} d(x, y)\right\}, \quad \forall A, B \in \mathscr{C}(X),
$$

where $d$ is a metric induced by the norm $\|\cdot\|_{X}$ of $X$.

Lemma 2.1 ( [20]). Let $A, B \in \mathscr{C}(X)$. For every $x \in A$, there exists $y \in B$ such that $d(x, y) \leq$ $\mathscr{H}(A, B)$.

We next recall the Riemann-Liouville fractional integral with order $\alpha>0$ of a suitable function $x$ (e.g., $x \in L^{1}\left(\left[t_{0}, t_{1}\right], \mathbf{R}\right)$ ) formulated below (see [21])

$$
I_{t_{0}}^{\alpha} x(t)=\frac{1}{\Gamma(\alpha)} \int_{t_{0}}^{t}(t-\tau)^{\alpha-1} x(\tau) d \tau, \quad\left(t>t_{0}\right),
$$

where $t_{0} \in \mathbf{R}$ and $\Gamma$ is the Gamma function. It is well known that $\Gamma(\alpha+1)=\alpha \Gamma(\alpha)(\alpha>0)$ and $\Gamma(1)=1$. Also, for a suitable function $x$ given on the interval $\left[t_{0}, t_{1}\right]$, we recall the Caputo fractional order derivative of order $\alpha$ of $x$ formulated below (see [21])

$$
{ }_{t_{0}}^{C} D_{t}^{\alpha} x(t)=I_{t_{0}}^{n-\alpha} x^{(n)}(t)= \begin{cases}\frac{1}{\Gamma(n-\alpha)} \int_{t_{0}}^{t}(t-\tau)^{n-\alpha-1} x^{(n)}(\tau) d \tau, & n-1<\alpha<n, \\ x^{(n)}(t), & \alpha=n,\end{cases}
$$

where $t>t_{0}$ and $n$ is a positive integer.

It is remarkable that if $x$ is an abstract function with values in a Banach space $X$, then the integrals which appear in the above definitions are taken in Bochner's sense. Let $T: X \rightarrow X$ be a nonlinear operator on $X$. $T$ is said to be nonexpansive if $\|T x-T y\|_{X} \leq\|x-y\|_{X}, \forall x, y \in X$. In particular, we provide an example of nonexpansive operators. Suppose that $K$ is a nonempty, closed, and convex subset of a real Hilbert space $H$. Then the projection operator $P_{K}: H \rightarrow K$ is nonexpansive.

Definition 2.1. Let $N_{1}: V_{1} \times V_{2} \rightarrow V_{1}$ be a nonlinear operator and $\Phi_{1}^{1}: V_{1} \rightarrow \mathscr{C}\left(V_{1}\right)$ be a setvalued mapping. The operator $N_{1}(\cdot, \cdot)$ is said to be $k_{1,1}$-strongly monotone for $\Phi_{1}^{1}$ with respect to the first argument if, for any $x_{1,1}, x_{1,2} \in V_{1}$,

$\left\langle N_{1}\left(w_{1,1}, \cdot\right)-N_{1}\left(w_{1,2}, \cdot\right), x_{1,1}-x_{1,2}\right\rangle_{V_{1}} \geq k_{1,1}\left\|x_{1,1}-x_{1,2}\right\|_{V_{1}}^{2}, \forall w_{1,1} \in \Phi_{1}^{1}\left(x_{1,1}\right), w_{1,2} \in \Phi_{1}^{1}\left(x_{1,2}\right)$.

Similarly, we can define the $k_{2,2}$-strong monotonicity of $N_{2}(\cdot, \cdot)$ for $\Phi_{2}^{2}$ with respect to the second argument.

Definition 2.2. Let $N_{1}: V_{1} \times V_{2} \rightarrow V_{1}$ be a nonlinear operator. The operator $N_{1}(\cdot, \cdot)$ is said to be $c_{1,1}$-Lipschitz with respect to the first argument if, for any $x_{1,1}, x_{1,2} \in V_{1}$,

$$
\left\|N_{1}\left(x_{1,1}, \cdot\right)-N_{1}\left(x_{1,2}, \cdot\right)\right\|_{V_{1}} \leq c_{1,1}\left\|x_{1,1}-x_{1,2}\right\|_{V_{1}} .
$$


Similarly, we can define the $l_{1,2}$-Lipschitz continuity of $N_{1}(\cdot, \cdot)$ with respect to the second argument.

Let $(\Omega, d)$ be a metric space, and let $T: \Omega \rightarrow 2^{\Omega}$ be a set-valued mapping with closed values. We say that $T$ is $\omega$-Lipschitz continuous if there exists a constant $\omega>0$ such that

$$
\mathscr{H}(T(x), T(y)) \leq \omega d(x, y), \quad \forall x, y \in \Omega .
$$

In particular, if $\omega<1$, then $T$ is called a set-valued $\omega$-contraction.

Definition 2.3 ( [22]). Let $Z$ and $Z_{1}$ be two topological spaces. A set-valued mapping $G: Z \rightarrow$ $2^{Z_{1}}$ is said to be

(i) upper semicontinuous (u.s.c.) if, for every open $O \subset Z_{1}, G^{-1}(O)=\{u \in Z: G(u) \subset O\}$ is open in $Z$;

(ii) lower semicontinuous (l.s.c.) if, for every closed $C \subset Z_{1}, G^{-1}(C)$ is closed in $Z$;

(iii) continuous if it is both u.s.c. and l.s.c.

Definition 2.4 ( [22]). Let $(\Omega, \Sigma)$ be a measurable space, and let $\Omega_{*}$ be a separable metric space. A set-valued mapping $G: \Omega \rightarrow 2^{\Omega_{*}}$ with closed values is said to be measurable if $G^{-1}(U) \in \Sigma$ for every open $U \subset \Omega_{*}$.

Definition 2.5. A pair of functions $\left(x_{1}, x_{2}\right) \in C\left(J, V_{1} \times V_{2}\right)$ is a solution of system (1.5) if there exists a pair of functions $\left(z_{1}, z_{2}\right) \in L^{1}\left(J, V_{1} \times V_{2}\right)$ such that

$$
\left\{\begin{array}{l}
z_{1}(t) \in P_{1}\left(x_{1}(t)-\rho_{1} N_{1}\left(\Phi_{1}^{1}\left(x_{1}(t)\right), \Phi_{2}^{1}\left(x_{2}(t)\right)\right)-\rho_{1} q_{1}\right)-x_{1}(t)+G_{1}\left(x_{1}(t)\right), \text { a.e. } t \in J, \\
z_{2}(t) \in P_{2}\left(x_{2}(t)-\rho_{2} N_{2}\left(\Phi_{1}^{2}\left(x_{1}(t)\right), \Phi_{2}^{2}\left(x_{2}(t)\right)\right)-\rho_{2} q_{2}\right)-x_{2}(t)+G_{2}\left(x_{2}(t)\right), \text { a.e. } t \in J,
\end{array}\right.
$$

and

$$
\left\{\begin{array}{l}
x_{1}(t)=x_{1,0}+\frac{1}{\Gamma(\alpha)} \int_{0}^{t}(t-\tau)^{\alpha-1} z_{1}(\tau) d \tau \\
x_{2}(t)=x_{2,0}+\frac{1}{\Gamma(\alpha)} \int_{0}^{t}(t-\tau)^{\alpha-1} z_{2}(\tau) d \tau .
\end{array}\right.
$$

Lemma 2.2 ( [22]). Let $\Omega_{*}$ be a separable complete metric space. Then every measurable $G: J=[0, b] \rightarrow 2^{\Omega_{*}}$ has a (single-valued) selection.

Lemma 2.3 ( [22]). Let $Z, Z_{1}$ be two topological spaces, and let $T: Z \rightarrow 2^{Z_{1}}$ be u.s.c. with compact values. Then $T(B)=\bigcup_{x \in B} T(x)$ is compact in $Z_{1}$ for any compact subset $B$ of $Z$.

Lemma 2.4 ( [3]). Let $T_{1}, T_{2}: J \rightarrow \mathscr{C}(X)$ be two continuous mappings in the Hausdorff metric $\mathscr{H}$, and let $f: J \rightarrow X$ be a measurable selection of $T_{1}$. Then there exists a measurable selection $g: J \rightarrow X$ of $T_{2}$ such that, for all $s \in J$,

$$
\|f(s)-g(s)\|_{X} \leq \mathscr{H}\left(T_{1}(s), T_{2}(s)\right) .
$$

Lemma 2.5 ( [23]). Let $X$ be a Banach space. The function $r: J=[0, b] \rightarrow X$ is Bochner integrable if and only if $r(\cdot)$ is strongly measurable and $\|r(\cdot)\|_{X}$ is Lebesgue integrable in J.

Lemma 2.6 ( [20]). Let $(\Omega, d)$ be a complete metric space. If $T: \Omega \rightarrow 2^{\Omega}$ is a set-valued contraction mapping with closed and bounded values, then $T$ has a fixed point in $\Omega$.

Lemma 2.7 ( [24]). Let $(\Omega, d)$ be a complete metric space, and let $T_{1}, T_{2}: \Omega \rightarrow 2^{\Omega}$ be two set-valued mappings with closed values. Assume that $T_{1}, T_{2}$ have the same contractive constant $\theta \in(0,1)$. Then

$$
\mathscr{H}\left(F\left(T_{1}\right), F\left(T_{2}\right)\right) \leq \frac{1}{1-\theta} \sup _{u \in \Omega} \mathscr{H}\left(T_{1}(u), T_{2}(u)\right),
$$

where $F\left(T_{1}\right)$ and $F\left(T_{2}\right)$ are fixed-point sets of $T_{1}$ and $T_{2}$, respectively. 


\section{MAIN RESULTS}

In this section, we study the existence of solutions and the sensitivity of the set of solutions for dynamical system (1.5) under some suitable conditions. Let $S\left(x_{1,0}, x_{2,0}\right)$ denote the set of all solutions for system (1.5) on $J=[0, b]$ with initial value $\left(x_{1,0}, x_{2,0}\right)$.

For a continuous mapping $A_{1}\left(x_{1}(\cdot)\right): J \rightarrow \mathscr{C}\left(V_{1}\right)$ and $x_{1} \in C\left(J, V_{1}\right)$, we define

$$
S_{A_{1}, x_{1}}=\left\{z_{1}(s) \in L^{1}\left(J, V_{1}\right): z_{1}(s) \in A_{1}\left(x_{1}(s)\right) \text { for a.e. } s \in J\right\} .
$$

Theorem 3.1. Let $G_{1}: V_{1} \rightarrow \mathscr{C}\left(V_{1}\right)$ be a $\omega_{1}$-Lipschitz continuous mapping, and let $G_{2}: V_{2} \rightarrow$ $\mathscr{C}\left(V_{2}\right)$ be a $\omega_{2}$-Lipschitz continuous mapping. Assume that

(i) $N_{1}: V_{1} \times V_{2} \rightarrow V_{1}$ is $k_{1,1}$-strongly monotone for $\Phi_{1}^{1}$ with respect to the first argument, $c_{1,1}$-Lipschitz with respect to the first argument and $l_{1,2}$-Lipschitz with respect to the second argument;

(ii) $\Phi_{1}^{1}: V_{1} \rightarrow \mathscr{C}\left(V_{1}\right)$ is $\beta_{1,1}$-Lipschitz continuous, and $\Phi_{2}^{1}: V_{2} \rightarrow \mathscr{C}\left(V_{2}\right)$ is $\beta_{1,2}$-Lipschitz continuous;

(iii) $N_{2}: V_{1} \times V_{2} \rightarrow V_{2}$ be $k_{2,2}$-strongly monotone for $\Phi_{2}^{2}$ with respect to the second argument, $c_{2,2}$-Lipschitz with respect to the second argument, and $l_{2,1}$-Lipschitz with respect to the first argument;

(iv) $\Phi_{1}^{2}: V_{1} \rightarrow \mathscr{C}\left(V_{1}\right)$ is $\beta_{2,1}$-Lipschitz continuous, and $\Phi_{2}^{2}: V_{2} \rightarrow \mathscr{C}\left(V_{2}\right)$ is $\beta_{2,2}$-Lipschitz continuous.

If

$$
\left\{\begin{array}{l}
\theta_{1}=\frac{b^{\alpha}}{\Gamma(\alpha+1)}\left(\sqrt{\xi}+\sqrt{\eta}+\omega_{1}+1\right)<1 \\
\theta_{2}=\frac{b^{\alpha}}{\Gamma(\alpha+1)}\left(\sqrt{\xi}+\sqrt{\eta}+\omega_{2}+1\right)<1
\end{array}\right.
$$

where

$$
\left\{\begin{array}{l}
\xi=\max \left\{1-2 \rho_{1} k_{1,1}+\rho_{1}^{2} c_{1,1}^{2} \beta_{1,1}^{2}, \rho_{1} l_{1,2} \beta_{1,2}+\rho_{1}^{2} c_{1,1} l_{1,2} \beta_{1,1} \beta_{1,2}, \rho_{1}^{2} l_{1,2}^{2} \beta_{1,2}^{2}\right\}>0 \\
\eta=\max \left\{1-2 \rho_{2} k_{2,2}+\rho_{2}^{2} c_{2,2}^{2} \beta_{2,2}^{2}, \rho_{2} l_{2,1} \beta_{2,1}+\rho_{2}^{2} c_{2,2} l_{2,1} \beta_{2,2} \beta_{2,1}, \rho_{2}^{2} l_{2,1}^{2} \beta_{2,1}^{2}\right\}>0
\end{array}\right.
$$

then $S\left(x_{1,0}, x_{2,0}\right)$ is nonempty, closed, and continuous with respect to the initial value $\left(x_{1,0}, x_{2,0}\right)$ in the sense of the Hausdorff metric.

Proof. For any $\left(x_{1}, x_{2}\right) \in V_{1} \times V_{2}$, let

$$
A_{1}\left(x_{1}, x_{2}\right)=P_{1}\left(x_{1}-\rho_{1} N_{1}\left(\Phi_{1}^{1}\left(x_{1}\right), \Phi_{2}^{1}\left(x_{2}\right)\right)-\rho_{1} q_{1}\right)-x_{1}+G_{1}\left(x_{1}\right) .
$$

Since $N_{1}: V_{1} \times V_{2} \rightarrow V_{1}, G_{1}, \Phi_{1}^{1}: V_{1} \rightarrow \mathscr{C}\left(V_{1}\right)$, and $\Phi_{2}^{1}: V_{2} \rightarrow \mathscr{C}\left(V_{2}\right)$ are continuous mappings, we know that $A_{1}: V_{1} \times V_{2} \rightarrow \mathscr{C}\left(V_{1}\right)$ is also a continuous mapping. For any $\left(x_{1,1}, x_{2,1}\right),\left(x_{1,2}, x_{2,2}\right) \in$ $V_{1} \times V_{2}$ and any $u_{1}^{1} \in A_{1}\left(x_{1,1}, x_{2,1}\right)$, there exist $\left(w_{1,1}, w_{2,1}\right) \in \Phi_{1}^{1}\left(x_{1,1}\right) \times \Phi_{2}^{1}\left(x_{2,1}\right)$ and $v_{1}^{1} \in$ $G_{1}\left(x_{1,1}\right)$ such that

$$
u_{1}^{1}=P_{1}\left(x_{1,1}-\rho_{1} N_{1}\left(w_{1,1}, w_{2,1}\right)-\rho_{1} q_{1}\right)-x_{1,1}+v_{1}^{1} .
$$

Since $\left(w_{1,1}, w_{2,1}\right) \in \Phi_{1}^{1}\left(x_{1,1}\right) \times \Phi_{2}^{1}\left(x_{2,1}\right), \Phi_{1}^{1}: V_{1} \rightarrow \mathscr{C}\left(V_{1}\right)$ is $\beta_{1,1}$-Lipschitz continuous, $\Phi_{2}^{1}$ : $V_{2} \rightarrow \mathscr{C}\left(V_{2}\right)$ is $\beta_{1,2}$-Lipschitz continuous and $G_{1}: V_{1} \rightarrow \mathscr{C}\left(V_{1}\right)$ is $\omega_{1}$-Lipschitz continuous, it follows from Lemma 2.1 that there exist $\left(w_{1,2}, w_{2,2}\right) \in \Phi_{1}^{1}\left(x_{1,2}\right) \times \Phi_{2}^{1}\left(x_{2,2}\right)$ and $v_{2}^{1} \in G_{2}\left(x_{1,2}\right)$ such that

$$
\begin{aligned}
& \left\|w_{1,1}-w_{1,2}\right\|_{V_{1}} \leq \mathscr{H}\left(\Phi_{1}^{1}\left(x_{1,1}\right), \Phi_{1}^{1}\left(x_{1,2}\right)\right) \leq \beta_{1,1}\left\|x_{1,1}-x_{1,2}\right\|_{V_{1}} \\
& \left\|w_{2,1}-w_{2,2}\right\|_{V_{2}} \leq \mathscr{H}\left(\Phi_{2}^{1}\left(x_{2,1}\right), \Phi_{2}^{1}\left(x_{2,2}\right)\right) \leq \beta_{1,2}\left\|x_{2,1}-x_{2,2}\right\|_{V_{2}}
\end{aligned}
$$


and

$$
\left\|v_{1}^{1}-v_{2}^{1}\right\|_{V_{1}} \leq \mathscr{H}\left(G_{1}\left(x_{1,1}\right), G_{1}\left(x_{1,2}\right)\right) \leq \omega_{1}\left\|x_{1,1}-x_{1,2}\right\|_{V_{1}}
$$

Let

$$
u_{2}^{1}=P_{1}\left(x_{1,2}-\rho_{1} N_{1}\left(w_{1,2}, w_{2,2}\right)-\rho_{1} q_{1}\right)-x_{1,2}+v_{2}^{1}
$$

Then $u_{2}^{1} \in A_{1}\left(x_{1,2}, x_{2,2}\right)$. Moreover, in light of (3.2)-(3.6) and the definition of nonexpansive operators, one has

$$
\begin{aligned}
\left\|u_{1}^{1}-u_{2}^{1}\right\|_{V_{1}} & =\|\left(P_{1}\left(x_{1,1}-\rho_{1} N_{1}\left(w_{1,1}, w_{2,1}\right)-\rho_{1} q_{1}\right)-x_{1,1}+v_{1}^{1}\right) \\
& -\left(P_{1}\left(x_{1,2}-\rho_{1} N_{1}\left(w_{1,2}, w_{2,2}\right)-\rho_{1} q_{1}\right)-x_{1,2}+v_{2}^{1}\right) \|_{V_{1}} \\
& \leq \| P_{1}\left(x_{1,1}-\rho_{1} N_{1}\left(w_{1,1}, w_{2,1}\right)-\rho_{1} q_{1}\right) \\
& -P_{1}\left(x_{1,2}-\rho_{1} N_{1}\left(w_{1,2}, w_{2,2}\right)-\rho_{1} q_{1}\right)\left\|_{V_{1}}+\right\| x_{1,1}-x_{1,2}\left\|_{V_{1}}+\right\| v_{1}^{1}-v_{2}^{1} \|_{V_{1}} \\
& \leq\left\|\left(x_{1,1}-x_{1,2}\right)-\rho_{1}\left(N_{1}\left(w_{1,1}, w_{2,1}\right)-N_{1}\left(w_{1,2}, w_{2,2}\right)\right)\right\|_{V_{1}} \\
& +\left(1+\omega_{1}\right)\left\|x_{1,1}-x_{1,2}\right\|_{V_{1}}
\end{aligned}
$$

and

$$
\begin{aligned}
& \left\|\left(x_{1,1}-x_{1,2}\right)-\rho_{1}\left(N_{1}\left(w_{1,1}, w_{2,1}\right)-N_{1}\left(w_{1,2}, w_{2,2}\right)\right)\right\|_{V_{1}}^{2} \\
& =\left\|x_{1,1}-x_{1,2}\right\|_{V_{1}}^{2}-2 \rho_{1}\left\langle N_{1}\left(w_{1,1}, w_{2,1}\right)-N_{1}\left(w_{1,2}, w_{2,2}\right), x_{1,1}-x_{1,2}\right\rangle_{V_{1}} \\
& +\rho_{1}^{2}\left\|N_{1}\left(w_{1,1}, w_{2,1}\right)-N_{1}\left(w_{1,2}, w_{2,2}\right)\right\|_{V_{1}}^{2} .
\end{aligned}
$$

Since $N_{1}: V_{1} \times V_{2} \rightarrow V_{1}$ is $k_{1,1}$-strongly monotone for $\Phi_{1}^{1}$ with respect to the first argument, $c_{1,1}$-Lipschitz with respect to the first argument and $l_{1,2}$-Lipschitz with respect to the second argument, we obtain from (3.3) and (3.4) that

$$
\begin{aligned}
& -2 \rho_{1}\left\langle N_{1}\left(w_{1,1}, w_{2,1}\right)-N_{1}\left(w_{1,2}, w_{2,2}\right), x_{1,1}-x_{1,2}\right\rangle_{V_{1}} \\
& =-2 \rho_{1}\left\langle N_{1}\left(w_{1,1}, w_{2,1}\right)-N_{1}\left(w_{1,2}, w_{2,1}\right)+N_{1}\left(w_{1,2}, w_{2,1}\right)-N_{1}\left(w_{1,2}, w_{2,2}\right), x_{1,1}-x_{1,2}\right\rangle_{V_{1}} \\
& =-2 \rho_{1}\left\langle N_{1}\left(w_{1,1}, w_{2,1}\right)-N_{1}\left(w_{1,2}, w_{2,1}\right), x_{1,1}-x_{1,2}\right\rangle_{V_{1}} \\
& -2 \rho_{1}\left\langle N_{1}\left(w_{1,2}, w_{2,1}\right)-N_{1}\left(w_{1,2}, w_{2,2}\right), x_{1,1}-x_{1,2}\right\rangle_{V_{1}} \\
& \leq-2 \rho_{1} k_{1,1}\left\|x_{1,1}-x_{1,2}\right\|_{V_{1}}^{2}+2 \rho_{1}\left\|N_{1}\left(w_{1,2}, w_{2,1}\right)-N_{1}\left(w_{1,2}, w_{2,2}\right)\right\|_{V_{1}}\left\|x_{1,1}-x_{1,2}\right\|_{V_{1}} \\
& \leq-2 \rho_{1} k_{1,1}\left\|x_{1,1}-x_{1,2}\right\|_{V_{1}}^{2}+2 \rho_{1} l_{1,2}\left\|x_{1,1}-x_{1,2}\right\|_{V_{1}}\left\|w_{2,1}-w_{2,2}\right\|_{V_{2}} \\
& \leq-2 \rho_{1} k_{1,1}\left\|x_{1,1}-x_{1,2}\right\|_{V_{1}}^{2}+2 \rho_{1} l_{1,2} \beta_{1,2}\left\|x_{1,1}-x_{1,2}\right\|\left\|_{V_{1}}\right\| x_{2,1}-x_{2,2} \|_{V_{2}},
\end{aligned}
$$

and

$$
\begin{aligned}
& \rho_{1}^{2}\left\|N_{1}\left(w_{1,1}, w_{2,1}\right)-N_{1}\left(w_{1,2}, w_{2,2}\right)\right\|_{V_{1}}^{2} \\
& =\rho_{1}^{2}\left\|N_{1}\left(w_{1,1}, w_{2,1}\right)-N_{1}\left(w_{1,2}, w_{2,1}\right)+N_{1}\left(w_{1,2}, w_{2,1}\right)-N_{1}\left(w_{1,2}, w_{2,2}\right)\right\|_{V_{1}}^{2} \\
& \leq \rho_{1}^{2}\left\|N_{1}\left(w_{1,1}, w_{2,1}\right)-N_{1}\left(w_{1,2}, w_{2,1}\right)\right\|_{V_{1}}^{2}+2 \rho_{1}^{2}\left\|N_{1}\left(w_{1,1}, w_{2,1}\right)-N_{1}\left(w_{1,2}, w_{2,1}\right)\right\|_{V_{1}} \\
& \quad \times\left\|N_{1}\left(w_{1,2}, w_{2,1}\right)-N_{1}\left(w_{1,2}, w_{2,2}\right)\right\|_{V_{1}}+\rho_{1}^{2}\left\|N_{1}\left(w_{1,2}, w_{2,1}\right)-N_{1}\left(w_{1,2}, w_{2,2}\right)\right\|_{V_{1}}^{2} \\
& \leq \rho_{1}^{2} c_{1,1}^{2}\left\|w_{1,1}-w_{1,2}\right\|_{V_{1}}^{2}+2 \rho_{1}^{2} c_{1,1} l_{1,2}\left\|w_{1,1}-w_{1,2}\right\|_{V_{1}}\left\|w_{2,1}-w_{2,2}\right\|_{V_{2}} \\
& +\rho_{1}^{2} l_{1,2}^{2}\left\|w_{2,1}-w_{2,2}\right\|_{V_{2}}^{2} \\
& \leq \rho_{1}^{2} c_{1,1}^{2} \beta_{1,1}^{2}\left\|x_{1,1}-x_{1,2}\right\|_{V_{1}}^{2}+2 \rho_{1}^{2} c_{1,1} l_{1,2} \beta_{1,1} \beta_{1,2}\left\|x_{1,1}-x_{1,2}\right\|_{V_{1}}\left\|x_{2,1}-x_{2,2}\right\|_{V_{2}} \\
& +\rho_{1}^{2} l_{1,2}^{2} \beta_{1,2}^{2}\left\|x_{2,1}-x_{2,2}\right\|_{V_{2}}^{2} .
\end{aligned}
$$


It follows from (3.8)-(3.10) that

$$
\begin{aligned}
& \left\|\left(x_{1,1}-x_{1,2}\right)-\rho_{1}\left(N_{1}\left(w_{1,1}, w_{2,1}\right)-N_{1}\left(w_{1,2}, w_{2,2}\right)\right)\right\|_{V_{1}}^{2} \\
& \leq\left\|x_{1,1}-x_{1,2}\right\|_{V_{1}}^{2}-2 \rho_{1} k_{1,1}\left\|x_{1,1}-x_{1,2}\right\|_{V_{1}}^{2}+2 \rho_{1} l_{1,2} \beta_{1,2}\left\|x_{1,1}-x_{1,2}\right\|_{V_{1}}\left\|x_{2,1}-x_{2,2}\right\|_{V_{2}} \\
& +\rho_{1}^{2} c_{1,1}^{2} \beta_{1,1}^{2}\left\|x_{1,1}-x_{1,2}\right\|_{V_{1}}^{2}+2 \rho_{1}^{2} c_{1,1} l_{1,2} \beta_{1,1} \beta_{1,2}\left\|x_{1,1}-x_{1,2}\right\|_{V_{1}}\left\|x_{2,1}-x_{2,2}\right\|_{V_{2}} \\
& +\rho_{1}^{2} l_{1,2}^{2} \beta_{1,2}^{2}\left\|x_{2,1}-x_{2,2}\right\|_{V_{2}}^{2} \\
& =\left(1-2 \rho_{1} k_{1,1}+\rho_{1}^{2} c_{1,1}^{2} \beta_{1,1}^{2}\right)\left\|x_{1,1}-x_{1,2}\right\|_{V_{1}}^{2}+\rho_{1}^{2} l_{1,2}^{2} \beta_{1,2}^{2}\left\|x_{2,1}-x_{2,2}\right\|_{V_{2}}^{2} \\
& +2\left(\rho_{1} l_{1,2} \beta_{1,2}+\rho_{1}^{2} c_{1,1} l_{1,2} \beta_{1,1} \beta_{1,2}\right)\left\|x_{1,1}-x_{1,2}\right\|_{V_{1}}\left\|x_{2,1}-x_{2,2}\right\|_{V_{2}} \\
& \leq \xi\left(\left\|x_{1,1}-x_{1,2}\right\|_{V_{1}}^{2}+2\left\|x_{1,1}-x_{1,2}\right\|_{V_{1}}\left\|x_{2,1}-x_{2,2}\right\|_{V_{2}}+\left\|x_{2,1}-x_{2,2}\right\|_{V_{2}}^{2}\right) \\
& =\xi\left(\left\|x_{1,1}-x_{1,2}\right\|_{V_{1}}+\left\|x_{2,1}-x_{2,2}\right\|_{V_{2}}\right)^{2}
\end{aligned}
$$

and hence

$$
\begin{aligned}
& \left\|\left(x_{1,1}-x_{1,2}\right)-\rho_{1}\left(N_{1}\left(w_{1,1}, w_{2,1}\right)-N_{1}\left(w_{1,2}, w_{2,2}\right)\right)\right\|_{V_{1}} \\
& \leq \sqrt{\xi}\left(\left\|x_{1,1}-x_{1,2}\right\|_{V_{1}}+\left\|x_{2,1}-x_{2,2}\right\|_{V_{2}}\right) .
\end{aligned}
$$

Combining (3.7) and (3.11), we obtain

$$
\begin{aligned}
\left\|u_{1}^{1}-u_{2}^{1}\right\|_{V_{1}} & \leq \sqrt{\xi}\left(\left\|x_{1,1}-x_{1,2}\right\|_{V_{1}}+\left\|x_{2,1}-x_{2,2}\right\|_{V_{2}}\right)+\left(1+\omega_{1}\right)\left\|x_{1,1}-x_{1,2}\right\|_{V_{1}} \\
& =\left(\sqrt{\xi}+\omega_{1}+1\right)\left\|x_{1,1}-x_{1,2}\right\|_{V_{1}}+\sqrt{\xi}\left\|x_{2,1}-x_{2,2}\right\|_{V_{2}} .
\end{aligned}
$$

In view of the definition of metric $d$ and (3.12), we have

$$
\begin{aligned}
& d\left(u_{1}^{1}, A_{1}\left(x_{1,2}, x_{2,2}\right)\right)=\inf _{u_{2}^{1} \in A_{1}\left(x_{1,2}, x_{2,2}\right)}\left\|u_{1}^{1}-u_{2}^{1}\right\|_{V_{1}} \\
& \leq\left(\sqrt{\xi}+\omega_{1}+1\right)\left\|x_{1,1}-x_{1,2}\right\|_{V_{1}}+\sqrt{\xi}\left\|x_{2,1}-x_{2,2}\right\|_{V_{2}} .
\end{aligned}
$$

Since $u_{1}^{1} \in A_{1}\left(x_{1,1}, x_{2,1}\right)$ is arbitrary, it follows that

$$
\sup _{u_{1}^{1} \in A_{1}\left(x_{1,1}, x_{2,1}\right)} d\left(u_{1}^{1}, A_{1}\left(x_{1,2}, x_{2,2}\right)\right) \leq\left(\sqrt{\xi}+\omega_{1}+1\right)\left\|x_{1,1}-x_{1,2}\right\|_{V_{1}}+\sqrt{\xi}\left\|x_{2,1}-x_{2,2}\right\|_{V_{2}} .
$$

Similarly, we can prove

$$
\sup _{u_{2}^{1} \in A_{1}\left(x_{1,2}, x_{2,2}\right)} d\left(A_{1}\left(x_{1,1}, x_{2,1}\right), u_{2}^{1}\right) \leq\left(\sqrt{\xi}+\omega_{1}+1\right)\left\|x_{1,1}-x_{1,2}\right\|_{V_{1}}+\sqrt{\xi}\left\|x_{2,1}-x_{2,2}\right\|_{V_{2}} .
$$

It follows from (3.13) and (3.14) that

$$
\mathscr{H}\left(A_{1}\left(x_{1,1}, x_{2,1}\right), A_{1}\left(x_{1,2}, x_{2,2}\right)\right) \leq\left(\sqrt{\xi}+\omega_{1}+1\right)\left\|x_{1,1}-x_{1,2}\right\|_{V_{1}}+\sqrt{\xi}\left\|x_{2,1}-x_{2,2}\right\|_{V_{2}} .
$$

Let

$$
A_{2}\left(x_{1}, x_{2}\right)=P_{2}\left(x_{2}-\rho_{2} N_{2}\left(\Phi_{1}^{2}\left(x_{1}\right), \Phi_{2}^{2}\left(x_{2}\right)\right)-\rho_{2} q_{2}\right)-x_{2}+G_{2}\left(x_{2}\right) .
$$

Since $N_{2}: V_{1} \times V_{2} \rightarrow V_{2}, G_{2}, \Phi_{2}^{2}: V_{2} \rightarrow \mathscr{C}\left(V_{2}\right)$ and $\Phi_{1}^{2}: V_{1} \rightarrow \mathscr{C}\left(V_{1}\right)$ are continuous mappings, we know that $A_{2}: V_{1} \times V_{2} \rightarrow \mathscr{C}\left(V_{2}\right)$ is also a continuous mapping. Similarly to the proof of (3.15), we can show that

$$
\mathscr{H}\left(A_{2}\left(x_{1,1}, x_{2,1}\right), A_{2}\left(x_{1,2}, x_{2,2}\right)\right) \leq\left(\sqrt{\eta}+\omega_{2}+1\right)\left\|x_{2,1}-x_{2,2}\right\|_{V_{2}}+\sqrt{\eta}\left\|x_{1,1}-x_{1,2}\right\|_{V_{1}} .
$$

Define a mapping $W_{0}: C\left(J, V_{1} \times V_{2}\right) \rightarrow 2^{C\left(J, V_{1} \times V_{2}\right)}$ as follows:

$$
W_{0}\left(x_{1}, x_{2}\right)=\left(W_{0}^{1}\left(x_{1}, x_{2}\right), W_{0}^{2}\left(x_{1}, x_{2}\right)\right)=\left(Q_{1}\left(x_{1}, x_{2}, x_{1,0}\right), Q_{2}\left(x_{1}, x_{2}, x_{2,0}\right)\right) \text {, }
$$

where

$$
Q_{1}\left(x_{1}, x_{2}, x_{1,0}\right)=\left\{f_{1} \in C\left(J, V_{1}\right): f_{1}(t)=x_{1,0}+\frac{1}{\Gamma(\alpha)} \int_{0}^{t}(t-s)^{\alpha-1} z_{1}(s) d s, z_{1}(s) \in S_{A_{1}, x_{1}}\right\},
$$


and

$$
Q_{2}\left(x_{1}, x_{2}, x_{2,0}\right)=\left\{f_{2} \in C\left(J, V_{2}\right): f_{2}(t)=x_{2,0}+\frac{1}{\Gamma(\alpha)} \int_{0}^{t}(t-s)^{\alpha-1} z_{2}(s) d s, z_{2}(s) \in S_{A_{2}, x_{2}}\right\} .
$$

Then it is easy to see that the solution of system (1.5) is equivalent to the fixed point of the mapping $W_{0}$, i.e.,

$$
S\left(x_{1,0}, x_{2,0}\right)=F\left(W_{0}\right)=\left\{\left(x_{1}, x_{2}\right):\left(x_{1}, x_{2}\right) \in W_{0}\left(x_{1}, x_{2}\right)\right\} .
$$

Now we show that $W_{0}$ is a set-valued mapping with nonempty and compact values. We first show that $Q_{1}: C\left(J, V_{1} \times V_{2}\right) \rightarrow 2^{C\left(J, V_{1}\right)}$ is a set-valued mapping with nonempty and compact values. In fact, for any given $\left(x_{1}, x_{2}\right) \in C\left(J, V_{1} \times V_{2}\right)$, since $A_{1}: V_{1} \times V_{2} \rightarrow \mathscr{C}\left(V_{1}\right)$ is a continuous mapping, we know that $A_{1}\left(x_{1}(\cdot), x_{2}(\cdot)\right): J \rightarrow \mathscr{C}\left(V_{1}\right)$ is continuous and it is also measurable. It follows from the separability of $V_{1}$ and Lemma 2.2 that there exists a measurable selection $z_{1}(s) \in A_{1}\left(x_{1}(s), x_{2}(s)\right)$ for all $s \in J$. So, $\left\|z_{1}(s)\right\|_{V_{1}}$ is measurable. Now Lemma 2.3 implies that there exists a constant $k_{1}>0$ such that

$$
\sup \left\{\left\|z_{1}(s)\right\|_{V_{1}}: z_{1}(s) \in A_{1}\left(x_{1}(s), x_{2}(s)\right)\right\} \leq k_{1}, \quad \forall s \in J .
$$

Therefore we know that $\left\|z_{1}(s)\right\|_{V_{1}}$ is Lebesgue integrable on $J=[0, b]$. In light of Lemma 2.5, we obtain that $z_{1}(s)$ is Bochner integrable. It follows that $z_{1}(s) \in S_{A_{1}, x_{1}}$. So, $Q_{1}\left(x_{1}, x_{2}, x_{1,0}\right)$ is nonempty. Moreover, for any $t \in J$, since

$$
f_{1}(t)=x_{1,0}+\frac{1}{\Gamma(\alpha)} \int_{0}^{t}(t-s)^{\alpha-1} z_{1}(s) d s
$$

with $z_{1}(s) \in S_{A_{1}, x_{1}}$, we have

$$
\begin{aligned}
\left\|f_{1}\right\|_{C\left(J, V_{1}\right)} & =\left\|x_{1,0}+\frac{1}{\Gamma(\alpha)} \int_{0}^{t}(t-s)^{\alpha-1} z_{1}(s) d s\right\|_{C\left(J, V_{1}\right)} \\
& \leq\left\|x_{1,0}\right\|_{V_{1}}+\frac{k_{1}}{\Gamma(\alpha)} \max _{t \in J} \int_{0}^{t}(t-s)^{\alpha-1} d s \\
& =\left\|x_{1,0}\right\|_{V_{1}}+\frac{k_{1}}{\Gamma(\alpha)} \max _{t \in J} \frac{t^{\alpha}}{\alpha} \\
& =\left\|x_{1,0}\right\|_{V_{1}}+\frac{k_{1} b^{\alpha}}{\Gamma(\alpha+1)} .
\end{aligned}
$$

This implies that $Q_{1}\left(x_{1}, x_{2}, x_{1,0}\right)$ is uniformly bounded.

Next, we show that $Q_{1}\left(x_{1}, x_{2}, x_{1,0}\right)$ is equicontinuous. Indeed, let $0 \leq t_{1}<t_{2} \leq b$. Since

$$
f_{1}\left(t_{i}\right)=x_{1,0}+\frac{1}{\Gamma(\alpha)} \int_{0}^{t_{i}}\left(t_{i}-s\right)^{\alpha-1} z_{1}(s) d s, \quad i=1,2,
$$

with $z_{1}(s) \in S_{A_{1}, x_{1}}$, one has

$$
f_{1}\left(t_{2}\right)-f_{1}\left(t_{1}\right)=\frac{1}{\Gamma(\alpha)}\left\{\int_{t_{1}}^{t_{2}}\left(t_{2}-s\right)^{\alpha-1} z_{1}(s) d s+\int_{0}^{t_{1}}\left(\left(t_{2}-s\right)^{\alpha-1}-\left(t_{1}-s\right)^{\alpha-1}\right) z_{1}(s) d s\right\},
$$

and hence

$$
\begin{aligned}
\left\|f_{1}\left(t_{2}\right)-f_{1}\left(t_{1}\right)\right\|_{V_{1}} & \leq \frac{k_{1}}{\Gamma(\alpha)}\left\{\left|\int_{t_{1}}^{t_{2}}\left(t_{2}-s\right)^{\alpha-1} d s\right|+\left|\int_{0}^{t_{1}}\left(\left(t_{2}-s\right)^{\alpha-1}-\left(t_{1}-s\right)^{\alpha-1}\right) d s\right|\right\} \\
& =\frac{k_{1}}{\Gamma(\alpha+1)}\left\{\left(t_{2}-t_{1}\right)^{\alpha}+\left|\left(t_{2}-t_{1}\right)^{\alpha}+t_{1}^{\alpha}-t_{2}^{\alpha}\right|\right\} \\
& \leq \frac{k_{1}}{\Gamma(\alpha+1)}\left(2\left(t_{2}-t_{1}\right)^{\alpha}+t_{1}^{\alpha}-t_{2}^{\alpha}\right) \leq \frac{2 k_{1}}{\Gamma(\alpha+1)}\left(t_{2}-t_{1}\right)^{\alpha}
\end{aligned}
$$

(due to the fact that $\left(a_{1}+a_{2}\right)^{\alpha} \leq a_{1}^{\alpha}+a_{2}^{\alpha}$ for all $a_{1}, a_{2} \geq 0$ with $0<\alpha \leq 1$ ). This shows that $Q_{1}\left(x_{1}, x_{2}, x_{1,0}\right)$ is equicontinuous. By Arzela-Ascoli Theorem, we deduce that $Q_{1}\left(x_{1}, x_{2}, x_{1,0}\right)$ is compact. 
In a similar way, we can prove that $Q_{2}: C\left(J, V_{1} \times V_{2}\right) \rightarrow 2^{C\left(J, V_{2}\right)}$ is a set-valued mapping with nonempty and compact values and so $W_{0}$ is a set-valued mapping with nonempty and compact values. Let

$$
W_{m}\left(x_{1}, x_{2}\right)=\left(W_{m}^{1}\left(x_{1}, x_{2}\right), W_{m}^{2}\left(x_{1}, x_{2}\right)\right)=\left(Q_{1}\left(x_{1}, x_{2}, x_{1,0}^{(m)}\right), Q_{2}\left(x_{1}, x_{2}, x_{2,0}^{(m)}\right)\right),
$$

where $\left(x_{1}, x_{2}\right) \in C\left(J, V_{1} \times V_{2}\right), m=1,2, \ldots$. Then

$$
W_{m}\left(x_{1}, x_{2}\right)=W_{0}\left(x_{1}, x_{2}\right)-\left(x_{1,0}, x_{2,0}\right)+\left(x_{1,0}^{(m)}, x_{2,0}^{(m)}\right)
$$

and $W_{m}: C\left(J, V_{1} \times V_{2}\right) \rightarrow \mathscr{C}\left(C\left(J, V_{1} \times V_{2}\right)\right)(m=0,1,2, \ldots)$ is a set-valued mapping. Let $\left(x_{1,0}^{(m)}, x_{2,0}^{(m)}\right)$ $\rightarrow\left(x_{1,0}, x_{2,0}\right)$ in $V=V_{1} \times V_{2}$ as $m \rightarrow \infty$, that is,

$$
\left.\|\left(x_{1,0}^{(m)}, x_{2,0}^{(m)}\right)\right)-\left(x_{1,0}, x_{2,0}\right)\left\|_{V}=\right\| x_{1,0}^{(m)}-x_{1,0}\left\|_{V_{1}}+\right\| x_{2,0}^{(m)}-x_{2,0} \|_{V_{2}} \rightarrow 0, \quad \text { as } m \rightarrow \infty .
$$

Then

$$
\mathscr{H}\left(W_{m}\left(x_{1}, x_{2}\right), W_{0}\left(x_{1}, x_{2}\right)\right) \rightarrow 0, \quad \text { as } m \rightarrow \infty .
$$

Next, we prove that $W_{m}: C\left(J, V_{1} \times V_{2}\right) \rightarrow \mathscr{C}\left(C\left(J, V_{1} \times V_{2}\right)\right), m=0,1,2, \ldots$, are set-valued contraction mappings. Indeed, for any $\left(x_{1,1}, x_{2,1}\right),\left(x_{1,2}, x_{2,2}\right) \in C\left(J, V_{1} \times V_{2}\right)$ and any $e_{1}^{1} \in$ $W_{m}^{1}\left(x_{1,1}, x_{2,1}\right)$, there exists $r_{1}^{1} \in S_{A_{1}, x_{1}}$ such that

$$
e_{1}^{1}(t)=x_{1,0}^{(m)}+\frac{1}{\Gamma(\alpha)} \int_{0}^{t}(t-s)^{\alpha-1} r_{1}^{1}(s) d s, \quad m=0,1,2, \ldots
$$

Since $r_{1}^{1}(s) \in A_{1}\left(x_{1,1}(s), x_{2,1}(s)\right)$ is Bochner integrable, it follows from Lemma 2.5 that $r_{1}^{1}(s)$ is measurable. Now Lemma 2.4 implies that there exists a measurable selection $r_{2}^{1}(s) \in A_{1}\left(x_{1,2}(s)\right.$, $\left.x_{2,2}(s)\right)$ such that, for all $s \in J=[0, b]$,

$$
\left\|r_{1}^{1}(s)-r_{2}^{1}(s)\right\|_{V_{1}} \leq \mathscr{H}\left(A_{1}\left(x_{1,1}(s), x_{2,1}(s)\right), A_{1}\left(x_{1,2}(s), x_{2,2}(s)\right)\right),
$$

and so $\left\|r_{2}^{1}(s)\right\|_{V_{1}}(s \in J)$ is measurable. By Lemma 2.3, we know that $\left\|r_{2}^{1}(s)\right\|_{V_{1}}(s \in J)$ is bounded. Hence $\left\|r_{2}^{1}(s)\right\|_{V_{1}}$ is Lebesgue integrable on $J$. Thus, by Lemma 2.5, we obtain that $r_{2}^{1}(s)$ is Bochner integrable. Let

$$
e_{2}^{1}(t)=x_{1,0}^{(m)}+\frac{1}{\Gamma(\alpha)} \int_{0}^{t}(t-s)^{\alpha-1} r_{2}^{1}(s) d s, \quad m=0,1,2, \ldots
$$

Then $e_{2}^{1} \in W_{m}^{1}\left(x_{1,2}, x_{2,2}\right)$. Furthermore, from (3.15) and (3.18), we have

$$
\begin{aligned}
& \left\|e_{1}^{1}-e_{2}^{1}\right\|_{C\left(J, V_{1}\right)} \\
& =\max _{t \in J} \frac{1}{\Gamma(\alpha)}\left\|\int_{0}^{t}(t-s)^{\alpha-1}\left(r_{1}^{1}(s)-r_{2}^{1}(s)\right) d s\right\|_{V_{1}} \\
& \leq \max _{t \in J} \frac{1}{\Gamma(\alpha)} \int_{0}^{t}(t-s)^{\alpha-1}\left\|r_{1}^{1}(s)-r_{2}^{1}(s)\right\|_{V_{1}} d s \\
& \leq \max _{t \in J} \frac{1}{\Gamma(\alpha)} \int_{0}^{t}(t-s)^{\alpha-1}\left(\left(\sqrt{\xi}+\omega_{1}+1\right)\left\|x_{1,1}(s)-x_{1,2}(s)\right\|_{V_{1}}+\sqrt{\xi}\left\|x_{2,1}(s)-x_{2,2}(s)\right\|_{V_{2}}\right) d s \\
& \leq \max _{t \in J} \frac{1}{\Gamma(\alpha)}\left(\left(\sqrt{\xi}+\omega_{1}+1\right)\left\|x_{1,1}-x_{1,2}\right\|_{C\left([0, t], V_{1}\right)}+\sqrt{\xi}\left\|x_{2,1}-x_{2,2}\right\|_{C\left([0, t], V_{2}\right)}\right) \int_{0}^{t}(t-s)^{\alpha-1} d s \\
& \leq \frac{b^{\alpha}}{\Gamma(\alpha+1)}\left(\left(\sqrt{\xi}+\omega_{1}+1\right)\left\|x_{1,1}-x_{1,2}\right\|_{C\left(J, V_{1}\right)}+\sqrt{\xi}\left\|x_{2,1}-x_{2,2}\right\|_{C\left(J, V_{2}\right)}\right) .
\end{aligned}
$$


It follows that

$$
\begin{aligned}
& \sup _{e_{1}^{1} \in W_{m}^{1}\left(x_{1,1}, x_{2,1}\right)} d\left(e_{1}^{1}, W_{m}^{1}\left(x_{1,2}, x_{2,2}\right)\right) \\
& =\sup _{e_{1}^{1} \in W_{m}^{1}\left(x_{1,1}, x_{2,1}\right)} \inf _{2}^{1} \in W_{m}^{1}\left(x_{1,2}, x_{2,2}\right) \\
& \leq \frac{b^{\alpha}}{\Gamma(\alpha+1)}\left(\left(\sqrt{\xi}+\omega_{1}^{1}+1\right)\left\|e_{2}^{1}\right\|_{C\left(J, V_{1}\right)}\right.
\end{aligned}
$$

Similarly, we can prove that

$$
\begin{aligned}
& \sup _{e_{2}^{1} \in W_{m}^{1}\left(x_{1,2}, x_{2,2}\right)} d\left(W_{m}^{1}\left(x_{1,1}, x_{2,1}\right), e_{2}^{1}\right) \\
& \leq \frac{b^{\alpha}}{\Gamma(\alpha+1)}\left(\left(\sqrt{\xi}+\omega_{1}+1\right)\left\|x_{1,1}-x_{1,2}\right\|_{C\left(J, V_{1}\right)}+\sqrt{\xi}\left\|x_{2,1}-x_{2,2}\right\|_{C\left(J, V_{2}\right)}\right) .
\end{aligned}
$$

By the definition of the Hausdorff metric, one has

$$
\begin{aligned}
& \mathscr{H}\left(W_{m}^{1}\left(x_{1,1}, x_{2,1}\right), W_{m}^{1}\left(x_{1,2}, x_{2,2}\right)\right) \\
& \leq \frac{b^{\alpha}}{\Gamma(\alpha+1)}\left(\left(\sqrt{\xi}+\omega_{1}+1\right)\left\|x_{1,1}-x_{1,2}\right\|_{C\left(J, V_{1}\right)}+\sqrt{\xi}\left\|x_{2,1}-x_{2,2}\right\|_{C\left(J, V_{2}\right)}\right)
\end{aligned}
$$

By the proof of (3.19), we can show that

$$
\begin{aligned}
& \mathscr{H}\left(W_{m}^{2}\left(x_{1,1}, x_{2,1}\right), W_{m}^{2}\left(x_{1,2}, x_{2,2}\right)\right) \\
& \leq \frac{b^{\alpha}}{\Gamma(\alpha+1)}\left(\left(\sqrt{\eta}+\omega_{2}+1\right)\left\|x_{2,1}-x_{2,2}\right\|_{C\left(J, V_{2}\right)}+\sqrt{\eta}\left\|x_{1,1}-x_{1,2}\right\|_{C\left(J, V_{1}\right)}\right) .
\end{aligned}
$$

In terms of (3.19) and (3.20), one has

$$
\begin{aligned}
& \mathscr{H}\left(W_{m}\left(x_{1,1}, x_{2,1}\right), W_{m}\left(x_{1,2}, x_{2,2}\right)\right) \\
& =\mathscr{H}\left(W_{m}^{1}\left(x_{1,1}, x_{2,1}\right), W_{m}^{1}\left(x_{1,2}, x_{2,2}\right)\right)+\mathscr{H}\left(W_{m}^{2}\left(x_{1,1}, x_{2,1}\right), W_{m}^{2}\left(x_{1,2}, x_{2,2}\right)\right) \\
& \leq \frac{b^{\alpha}}{\Gamma(\alpha+1)}\left(\left(\sqrt{\xi}+\omega_{1}+1\right)\left\|x_{1,1}-x_{1,2}\right\|_{C\left(J, V_{1}\right)}+\sqrt{\xi}\left\|x_{2,1}-x_{2,2}\right\|_{C\left(J, V_{2}\right)}\right) \\
& +\frac{b^{\alpha}}{\Gamma(\alpha+1)}\left(\left(\sqrt{\eta}+\omega_{2}+1\right)\left\|x_{2,1}-x_{2,2}\right\|_{C\left(J, V_{2}\right)}+\sqrt{\eta}\left\|x_{1,1}-x_{1,2}\right\|_{C\left(J, V_{1}\right)}\right) \\
& =\frac{b^{\alpha}}{\Gamma(\alpha+1)}\left(\left(\sqrt{\xi}+\sqrt{\eta}+\omega_{1}+1\right)\left\|x_{1,1}-x_{1,2}\right\|_{C\left(J, V_{1}\right)}\right. \\
& \left.+\left(\sqrt{\xi}+\sqrt{\eta}+\omega_{2}+1\right)\left\|x_{2,1}-x_{2,2}\right\|_{C\left(J, V_{2}\right)}\right) \\
& \leq \theta\left(\left\|x_{1,1}-x_{1,2}\right\|_{C\left(J, V_{1}\right)}+\left\|x_{2,1}-x_{2,2}\right\|_{C\left(J, V_{2}\right)}\right)
\end{aligned}
$$

where $\theta=\max \left\{\theta_{1}, \theta_{2}\right\}$ with $\theta_{i}=b^{\alpha}\left(\sqrt{\xi}+\sqrt{\eta}+\omega_{i}+1\right) / \Gamma(\alpha+1)$ for $i=1,2$. In terms of (3.1) and (3.21), we know that $W_{m}: C\left(J, V_{1} \times V_{2}\right) \rightarrow \mathscr{C}\left(C\left(J, V_{1} \times V_{2}\right)\right), m=0,1,2, \ldots$, are setvalued contractive mappings with the same contractive constant $\theta \in(0,1)$. From Lemma 2.6 it follows that $W_{0}\left(x_{1}, x_{2}\right)$ has a fixed point for each given $\left(x_{1,0}, x_{2,0}\right)$. So, $S\left(x_{1,0}, x_{2,0}\right)$ is nonempty for each given $\left(x_{1,0}, x_{2,0}\right)$.

In addition, we show that the solution set of system (1.5) is closed. Indeed, since the solution of system (1.5) is equivalent to the fixed point of the mapping $W_{0}\left(x_{1}, x_{2}\right)$, we only need to show that the fixed point set of $W_{0}\left(x_{1}, x_{2}\right)$ is closed. Let $\left\{\left(x_{1, n}, x_{2, n}\right)\right\} \subseteq F\left(W_{0}\right)$ with $\left(x_{1, n}, x_{2, n}\right) \rightarrow$ $\left(x_{1,0}, x_{2,0}\right)(n \rightarrow \infty)$ in $V=V_{1} \times V_{2}$. Then $\left(x_{1, n}, x_{2, n}\right) \in W_{0}\left(x_{1, n}, x_{2, n}\right)$. In view of (3.21), we have

$$
\mathscr{H}\left(W_{0}\left(x_{1, n}, x_{2, n}\right), W_{0}\left(x_{1,0}, x_{2,0}\right)\right) \leq \theta\left\|\left(x_{1, n}, x_{2, n}\right)-\left(x_{1,0}, x_{2,0}\right)\right\|_{V_{1} \times V_{2}}
$$

and

$$
\begin{aligned}
& d\left(\left(x_{1,0}, x_{2,0}\right), W_{0}\left(x_{1,0}, x_{2,0}\right)\right) \\
& \leq\left\|\left(x_{1,0}, x_{2,0}\right)-\left(x_{1, n}, x_{2, n}\right)\right\|_{V_{1} \times V_{2}} \\
& \quad+d\left(\left(x_{1, n}, x_{2, n}\right), W_{0}\left(x_{1, n}, x_{2, n}\right)\right)+\mathscr{H}\left(W_{0}\left(x_{1, n}, x_{2, n}\right), W_{0}\left(x_{1,0}, x_{2,0}\right)\right) \\
& \leq(1+\theta)\left\|\left(x_{1, n}, x_{2, n}\right)-\left(x_{1,0}, x_{2,0}\right)\right\|_{V_{1} \times V_{2}} \rightarrow 0 \quad(n \rightarrow \infty) .
\end{aligned}
$$

It follows that $\left(x_{1,0}, x_{2,0}\right) \in F\left(W_{0}\right)$ and so $F\left(W_{0}\right)$ is closed. 
Finally, we show that the set of solutions of (1.5) is continuous with respect to the initial value in the sense of the Hausdorff metric. Indeed, from Lemma 2.7, (3.17), and (3.21), we conclude that

$$
\mathscr{H}\left(F\left(W_{m}\right), F\left(W_{0}\right)\right) \leq \frac{1}{1-\theta} \sup _{\left(x_{1}, x_{2}\right) \in V_{1} \times V_{2}} \mathscr{H}\left(W_{m}\left(x_{1}, x_{2}\right), W_{0}\left(x_{1}, x_{2}\right)\right) \rightarrow 0, \quad \text { as } m \rightarrow \infty .
$$

Thus, $F\left(W_{m}\right) \rightarrow F\left(W_{0}\right)$ as $m \rightarrow \infty$, which implies that $S\left(x_{1,0}^{(m)}, x_{2,0}^{(m)}\right) \rightarrow S\left(x_{1,0}, x_{2,0}\right)$ as $m \rightarrow \infty$, that is, the set of solutions of system (1.5) is continuous with respect to initial value $\left(x_{1,0}, x_{2,0}\right)$ in the sense of the Hausdorff metric.

It is remarkable that Theorem 3.1 improves and extends [3, Theorem 3.1] from the fractionalorder projective dynamical systems involving set-valued perturbations to generalized fractionalorder composite dynamical systems involving set-valued perturbations in the same frameworks of real separable Hilbert spaces. However, [3, Theorem 3.1] improves and extends [16, Theorem 3.1] from the fractional-order projective dynamical systems in finite dimensional spaces to the fractional-order projective dynamical systems involving set-valued perturbations in real separable Hilbert spaces. In addition, [3, Theorem 3.1] can be considered as a generalization of the corresponding results of $[4,25]$ in the sense of fractional derivative. Therefore, Theorem 3.1 also improves and extends the corresponding results of $[4,16,25]$.

\section{An EXAMPLE}

In this section, we provide an example to illustrate that all the hypotheses in Theorem 3.1 can be satisfied.

Example 4.1. Suppose that $V_{1}=V_{2}=\mathbf{R}, q_{1}=-0.7, q_{2}=2, x_{1,0}=-1.2, x_{2,0}=3.4$ and $P_{1}=$ $P_{K_{1}}, P_{2}=P_{K_{2}}$ with $K_{1}=[-1,2], K_{2}=[3,5]$. We define $N_{1}(x, y)=2 x+y$ and $N_{2}(x, y)=x+2 y$ for all $(x, y) \in V_{1} \times V_{2}$. Let

$$
G_{1}(x)=\left\{\begin{array}{ll}
{[0,0.02 x], \quad x \geq 0,} \\
0, \quad x<0,
\end{array} \quad \text { and } \quad G_{2}(y)= \begin{cases}0, & y>0, \\
{[0,-0.03 y],} & y \leq 0 .\end{cases}\right.
$$

Moreover, we define $\Phi_{1}^{1}(x)=-0.015 \cos x+1.64 x, \Phi_{2}^{1}(y)=0.8 \sin y$,

$$
\Phi_{1}^{2}(x)=-0.3 \sin x \quad \text { and } \Phi_{2}^{2}(y)=-0.1 \arctan y+2.05 y,
$$

for all $(x, y) \in V_{1} \times V_{2}$. Then it is easy to see that

$$
\begin{aligned}
& \left|N_{1}\left(x^{1}, \cdot\right)-N_{1}\left(x^{2}, \cdot\right)\right| \leq 2\left|x^{1}-x^{2}\right|=c_{1,1}\left|x^{1}-x^{2}\right|, \\
& \left|N_{1}\left(\cdot, y^{1}\right)-N_{1}\left(\cdot, y^{2}\right)\right| \leq\left|y^{1}-y^{2}\right|=l_{1,2}\left|y^{1}-y^{2}\right|, \\
& \left(N_{1}\left(\Phi_{1}^{1}\left(x^{1}\right), \cdot\right)-N_{1}\left(\Phi_{1}^{1}\left(x^{2}\right), \cdot\right)\right)\left(x^{1}-x^{2}\right) \geq 3.25\left(x^{1}-x^{2}\right)^{2}=k_{1,1}\left(x^{1}-x^{2}\right)^{2}, \\
& \left|\Phi_{1}^{1}\left(x^{1}\right)-\Phi_{1}^{1}\left(x^{2}\right)\right| \leq 1.655\left|x^{1}-x^{2}\right|=\beta_{1,1}\left|x^{1}-x^{2}\right|, \\
& \left|\Phi_{2}^{1}\left(y^{1}\right)-\Phi_{2}^{1}\left(y^{2}\right)\right| \leq 0.8\left|y^{1}-y^{2}\right|=\beta_{1,2}\left|y^{1}-y^{2}\right| \\
& \left|N_{2}\left(\cdot, y^{1}\right)-N_{2}\left(\cdot, y^{2}\right)\right| \leq 2\left|y^{1}-y^{2}\right|=c_{2,2}\left|y^{1}-y^{2}\right| \\
& N_{2}\left(x^{1}, \cdot\right)-N_{2}\left(x^{2}, \cdot\right)|\leq| x^{1}-x^{2}\left|=l_{2,1}\right| x^{1}-x^{2} \mid \\
& \left(N_{2}\left(\cdot, \Phi_{2}^{2}\left(y^{1}\right)\right)-N_{2}\left(\cdot, \Phi_{2}^{2}\left(y^{2}\right)\right)\right)\left(y^{1}-y^{2}\right) \geq 3.9\left(y^{1}-y^{2}\right)^{2}=k_{2,2}\left(y^{1}-y^{2}\right)^{2}, \\
& \left|\Phi_{1}^{2}\left(x^{1}\right)-\Phi_{1}^{2}\left(x^{2}\right)\right| \leq 0.3\left|x^{1}-x^{2}\right|=\beta_{2,1}\left|x^{1}-x^{2}\right| \\
& \left|\Phi_{2}^{2}\left(y^{1}\right)-\Phi_{2}^{2}\left(y^{2}\right)\right| \leq 2.15\left|y^{1}-y^{2}\right|=\beta_{2,2}\left|y^{1}-y^{2}\right| \\
& \mathscr{H}\left(G_{1}\left(x^{1}\right), G_{1}\left(x^{2}\right)\right) \leq 0.02\left|x^{1}-x^{2}\right|=\omega_{1}\left|x^{1}-x^{2}\right| \\
& \mathscr{H}\left(G_{2}\left(y^{1}\right), G_{2}\left(y^{2}\right)\right) \leq 0.03\left|y^{1}-y^{2}\right|=\omega_{2}\left|y^{1}-y^{2}\right|
\end{aligned}
$$


for all $x^{1}, x^{2} \in V_{1}, y^{1}, y^{2} \in V_{2}$. If $\rho_{1}=0.16$ and $\rho_{2}=0.2$, then

$$
\begin{aligned}
\xi & =\max \left\{1-2 \rho_{1} k_{1,1}+\rho_{1}^{2} c_{1,1}^{2} \beta_{1,1}^{2}, \rho_{1} l_{1,2} \beta_{1,2}+\rho_{1}^{2} c_{1,1} l_{1,2} \beta_{1,1} \beta_{1,2}, \rho_{1}^{2} l_{1,2}^{2} \beta_{1,2}^{2}\right\} \\
& =\max \{0.2405,0.1958,0.0164\} \\
& =0.2405
\end{aligned}
$$

and

$$
\begin{aligned}
\eta & =\max \left\{1-2 \rho_{2} k_{2,2}+\rho_{2}^{2} c_{2,2}^{2} \beta_{2,2}^{2}, \rho_{2} l_{2,1} \beta_{2,1}+\rho_{2}^{2} c_{2,2} l_{2,1} \beta_{2,2} \beta_{2,1}, \rho_{2}^{2} l_{2,1}^{2} \beta_{2,1}^{2}\right\} \\
& =\max \{0.1796,0.1116,0.0036\} \\
& =0.1796 .
\end{aligned}
$$

Moreover, if $b=0.35, \alpha=0.8$, then

$$
\begin{aligned}
\theta_{1} & =\frac{b^{\alpha}}{\Gamma(\alpha+1)}\left(\sqrt{\xi}+\sqrt{\eta}+\omega_{1}+1\right) \\
& =\frac{0.4318}{0.9314}(0.4904+0.4238+0.02+1) \\
& =0.4636 \times 1.9342=0.8967<1
\end{aligned}
$$

and

$$
\begin{aligned}
\theta_{2} & =\frac{b^{\alpha}}{\Gamma(\alpha+1)}\left(\sqrt{\xi}+\sqrt{\eta}+\omega_{2}+1\right) \\
& =\frac{0.4318}{0.9314}(0.4904+0.4238+0.03+1) \\
& =0.4636 \times 1.9442=0.9013<1 .
\end{aligned}
$$

Therefore, it is easy to see that (3.1) holds and so the solution set of this system is nonempty, closed, and continuous with respect to initial value $\left(x_{1,0}, x_{2,0}\right)$ in the sense of the Hausdorff metric.

\section{The Concluding Remark}

In this paper, we studied, for the first time, the generalized fractional composite dynamical system with set-valued perturbations (i.e., (1.5)) in real separable Hilbert spaces. It is worth mentioning that if $P_{i}=I$ the identity mapping of $V_{i}$ for $i=1,2$, then (1.5) reduces to the system of two related generalized fractional differential inclusions with set-valued perturbations in abstract spaces. Model (1.5) captures the desired features of both composite dynamical systems and generalized fractional differential inclusions with set-valued perturbations within the same frameworks. Therefore, the generalized fractional composite dynamical system with set-valued perturbations is important and interesting in theory and practice. And also it is necessary to study the property of the solution set. Based on the fixed point theorem for set-valued contractive mappings, selection theorem, etc., we established a sufficient condition for the nonemptiness and closedness of the solutions set of system (1.5). Furthermore, we showed that the set of solutions is continuous with respect to initial value in the sense of the Hausdorff metric. In addition, a numerical example was provided to illustrate the main results obtained in this paper. Compared with the existing model of fractional projective dynamical systems with set-valued perturbations, model (1.5) is more general and more advantageous since it captures the desired features of both composite dynamical systems and generalized fractional differential inclusions with set-valued perturbations within the same framework. It is worth to point out that RiemannLiouville type fractional derivative has nice and useful mathematical properties. It was known in [26] that, under zeros initial conditions, the equations with Riemann-Liouville operators are equivalent to those with Caputo operators. So Theorem 3.1 holds under Riemann-Liouville type fractional derivative with zeros initial conditions. In general, does the Theorem 3.1 still hold 
under Riemann-Liouville type fractional derivative? We will consider such a problem in our future work.

\section{Acknowledgments}

The authors are grateful to the reviewers for useful suggestions which improved the contents of this paper. The first author was supported by the 2020 Shanghai Leading Talents Program of the Shanghai Municipal Human Resources and Social Security Bureau (20LJ2006100). The second author was supported by the National Natural Science Foundation of China (11671282). The third author was supported by the Ministry of Science and Technology, Taiwan under Grant No. 110-2115-M-037-001.

\section{REFERENCES}

[1] P. Dupuis, A. Nagurney, Dynamical systems and variational inequalities, Ann. Oper. Res. 44 (1993), 9-42.

[2] T.L. Friesz, D. Bernstein, N.J. Mehta, R.L. Tobin, S. Ganjalizadeh, Day-to-day dynamical network disequilibria and idealized traveler information systems, Oper. Res. 42 (1994), 1120-1136.

[3] Z.B. Wu, Y.Z. Zou, N.J. Huang, A class of global fractional-order projective dynamical systems involving set-valued perturbations, Appl. Math. Comput. 277 (2016), 23-33.

[4] Y.Z. Zou, C.Y. Sun, Equilibrium points for two related projective dynamical systems, Commun. Appl. Nonlinear Anal. 19 (2012) 109-117.

[5] L.V. Nguyen, X. Qin, Some results on strongly pseudomonotone quasi-variational inequalities, Set-Valued Var. Anal. 28 (2020), 239-257.

[6] L.V. Nguyen, Q.H. Ansari, X. Qin, Weak sharpness and finite convergence for solutions of nonsmooth variational inequalities in Hilbert spaces, Appl. Math. Optim. 84 (201), 807-828.

[7] S.Y. Cho, A monotone Bregan projection algorithm for fixed point and equilibrium problems in a reflexive Banach space, Filomat, 34 (2020), 1487-1497.

[8] L.C. Ceng, C.F. Wen, Systems of variational inequalities with hierarchical variational inequality constraints for asymptotically nonexpansive and pseudocontractive mappings, Rev. R. Acad. Cienc. Exactas Fís. Nat. Ser. A Mat. RACSAM 113 (2019), 2431-2447.

[9] C.Y. Wang, L.C. Ceng, L. He et al., On the parallel subgradient extragradient rule for solving systems of variational inequalities in Hadamard manifolds, Symmetry 13 (2021), Article ID 1496.

[10] L.C. Ceng, Z.R. Kong, C.F. Wen, On general systems of variational inequalities, Comput. Math. Appl. 66 (2013), 1514-1532.

[11] P.J. Torvik, R.L. Bagley, On the appearance of the fractional derivative in the behavior of real materials, J. Appl. Mech. 51 (1984), 284-298.

[12] C.P. Li, F.R. Zhang, A survey on the stability of fractional differential equations, Eur. Phys. J. Spec. Top. 193 (2011), 27-47.

[13] N. Ozalp, I. Koca, A fractional order nonlinear dynamical model of interpersonal relationships, Adv. Difference Equ. 2012 (2012), 189.

[14] F. Buyukkilic, Z. Bayrakdar, D. Demirhan, Investigation of cumulative growth process via Fibonacci method and fractional calculus, Appl. Math. Comput. 265 (2015), 237-244.

[15] O. Carja, T. Donchev, M. Rafaqat, R. Ahmed, Viability of fractional differential inclusions, Appl. Math. Lett. 38 (2014), 48-51.

[16] Z.B. Wu, Y.Z. Zou, Global fractional-order projective dynamical systems, Commun. Nonlinear Sci. Numer. Simul. 19 (2014), 2811-2819.

[17] X.K. Wu, Z.B. Wu, Y.Z. Zou, Sensitivity of the set of solutions for a class of fractional set-valued projected dynamical systems, Nonlinear Anal. Forum 20 (2015), 95-105.

[18] Z.B. Wu, Y.Z. Zou, N.J. Huang, A system of fractional-order interval projection neural networks, J. Comput. Appl. Math. 294 (2016), 389-402.

[19] E. Zeidler, Nonlinear Functional Analysis and Its Applications, Springer-Verlag, Berlin, 1990. 
[20] S.B. Nadler Jr., Multivalued contraction mappings, Pacific J. Math. 30 (1969), 475-488.

[21] A.A. Kilbas, H.M. Srivastava, J.J. Trujillo, Theory and Applications of Fractional Differential Equations, Elsevier, 2006.

[22] L. Gorniewicz, Topological Fixed Point Theory of Multivalued Mappings, 2ed., Springer, 2006.

[23] G.E. Ladas, V. Lakshmikantham, Differential Equations in Abstract Spaces, Academic Press, New York and London, 1972.

[24] T.C. Lim, On fixed point stability for set-valued contractive mappings with applications to generalized differential equations, J. Math. Anal. Appl. 110 (1985), 436-441.

[25] Z.B. Wu, Y.Z. Zou, Stability analysis of two related projective dynamical systems in Hilbert spaces, Nonlinear Anal. Forum 19 (2014), 37-51.

[26] K. Diethelm, The Analysis of Fractional Differential Equations, Springer, Berlin, 2010. 